Kennedy PJ, Clarke G, Quigley EM, Groeger JA, Dinan TG, Cryan JF (2012). Gut memories: towards a cognitive neurobiology of irritable bowel syndrome. Neurosci Biobehav Rev 36: 310-340.

Strober W, Fuss IJ, Blumberg RS (2002). The immunology of mucosal models of inflammation. Annu Rev Immunol 20: 495-549.

Zonis S, Ljubimov VA, Mahgerefteh M, Pechnick RN, Wawrowsky K, Chesnokova V (2013). p21Cip restrains hippocampal neurogenesis and protects neuronal progenitors from apoptosis during acute systemic inflammation. Hippocampus 23 1383-1394.

Zonis S, Pechnick RN, Ljubimov VA, Mahgerefteh M, Wawrowsky K, Michelsen KS et al (2015). Chronic intestinal inflammation alters hippocampal neurogenesis. J Neuroinflammation 12: 65.

Neuropsychopharmacology Reviews (2016) 41, 372-373 doi: I 0.1038/npp.2015.237

\section{Neural Basis of Mindfulness Interventions that Moderate the Impact of Stress on the Brain}

The scientific study of mindfulness has skyrocketed. Mindfulness can be defined as 'non-judgmental attention to present-moment experiences' and is thought to comprise several complex processes, including attentional control, emotion regulation, and selfawareness (Tang et al, 2015). Although the neuroscience underlying mindfulness is at an early stage, there are some intriguing findings that begin to unravel the effects of mindfulness on mental health, stress, and resilience. For example, those individuals who rated themselves as more mindful, i.e. had greater 'dispositional mindfulness', generally report lower levels of perceived stress (Prakash et al, 2015). This is important because the level of stress is strongly related to physical and mental health as well as cortical thinning. In comparison, dispositional mindfulness has been related to structural and functional differences in several neural structures, including the medial prefrontal cortex, hippocampus, amygdala, anterior and posterior cingulate, and orbitofrontal cortex (Tang et al, 2015). Therefore, dispositional mindfulness may prove to be an important construct to examine individual differences that can help to predict risk for and relapse to mental disorders.

Mindfulness-based stress reduction (MBSR) has been proposed for almost every psychiatric condition. In a meta-analysis (Sedlmeier et al, 2012), mindfulness interventions had medium to large effect sizes for changes in emotionality and relationship issues, medium effect sizes for measures of attention, and small effect sizes for cognitive measures. MBSR has been associated with increased cortical thickness in the insula and somatosensory cortex, which can be associated with reduction of worry, state anxiety, depression, and alexithymia (Tang et al, 2015). Moreover, changes after mindfulness training in the insula have been related to increase in interoceptive awareness, i.e. the ability to monitor afferents from inside the body, which is emerging as an important construct for anxiety disorders and addiction (Paulus and Stewart, 2013). Thus, some of the same brain systems that have been implicated in dispositional mindfulness are also affected by mindfulness-based interventions and show a certain degree of plasticity of these systems.

Our understanding of the molecular mechanisms of mindfulness and changes induced by mindfulnessbased interventions is at its infancy. Recent studies have reported that MBSR training results in a smaller post-stress inflammatory response (Rosenkranz et al, 2013), which includes interleukin-6. MBSR also increased telomerase activity and those individuals with the greatest increase also reported the greatest reductions in chronic stress, anxiety, dietary restraint, dietary fat intake, cortisol, and glucose (Daubenmier et al, 2012). These findings suggest that mindfulness interventions affect both inflammatory and epigenetic mechanisms, which are important for mood and stress-related disorders, respectively. Therefore, elucidation of the molecular substrates that underlie individual differences in mindfulness may be one of the most fruitful areas for future research. Taken together, mindfulness and mindfulness-based interventions have profound effects on mental health, affect brain systems that are important for emotion regulation and self-awareness, and alter inflammatory and epigenetic responses, yet much needs to be done to make these interventions a part of precision psychiatry.

\section{FUNDING AND DISCLOSURE}

The author declares no conflict of interest.

\section{ACKNOWLEDGMENTS}

This study was funded by the William K Warren Foundation.

\section{Martin P Paulus ${ }^{1}$}

${ }^{1}$ Laureate Institute for Brain Research, Tulsa, OK, USA E-mail: mpaulus@laureateinstitute.org

Daubenmier J, Lin J, Blackburn E, Hecht FM, Kristeller J, Maninger N et al (2012). Changes in stress, eating, and metabolic factors are related to changes in telomerase activity in a randomized mindfulness intervention pilot study. Psychoneuroendocrinology 37: 917-928.

Paulus MP, Stewart JL (2013). Interoception and drug addiction. Neuropharmacology 76(Pt B): 342-350.

Prakash RS, Hussain MA, Schirda B (2015). The role of emotion regulation and cognitive control in the association between mindfulness disposition and stress. Psychol Aging 30: 160-171.

Rosenkranz MA, Davidson RJ, Maccoon DG, Sheridan JF, Kalin NH, Lutz A (2013). A comparison of mindfulness-based stress reduction and an active control in modulation of neurogenic inflammation. Brain Behav Immun 27: 174-184.

Sedlmeier P, Eberth J, Schwarz M, Zimmermann D, Haarig F, Jaeger S et al (2012). The psychological effects of meditation: a meta-analysis. Psychol Bull 138: 1139-1171.

Tang YY, Holzel BK, Posner Ml (2015). The neuroscience of mindfulness meditation. Nat Rev Neurosci 16: 213-225.

Neuropsychopharmacology Reviews (2016) 41, 373; doi: 10.1038/npp.2015.239

\section{Dynorphin, Dysphoria, and Dependence: the Stress of Addiction}

The hypothesis that the dynorphinkappa opioid receptor system may be a key component of the neuroplasticity associated with stress-induced mood disorders and the 'dark side' of 
addiction (withdrawal-negative affect stage) continues to gain preclinical and clinical experimental support. The endogenous kappa opioid peptides derived from prodynorphin encode the dysphoric, anxiogenic, and cognitive disrupting responses to behavioral stress exposure (Bruchas et al, 2010; Carroll and Carlezon, 2013). Drugs of abuse are also profound activators of the brain stress systems, and dynorphin release following a binge of consumption contributes to the dysphoric and anhedonic responses experienced during withdrawal (Koob et al, 2014). Behavioral studies using rodents in multiple laboratories have now consistently demonstrated that kappa antagonists do not block the 'euphoric-like' effects of drugs but rather block the stress-induced potentiation of drug reward, block stress-induced reinstatement of drug seeking behavior, and block escalation of drug consumption in long-access models (Whitfield et al, 2015). We predict that kappa antagonists will promote stress resilience and disrupt the addiction cycle by reducing the dysphoria-driven cravings that trigger a subsequent round of drug seeking.

However, very exciting preclinical findings too often fail to deliver on their promises, particularly in CNS drug development, which is notoriously expensive and difficult. Progress is being made with a kappa antagonist (LY2456302) developed by Eli Lilly scientists, which passed initial safety testing and has been licensed for development by Cerecor (Lowe et al, 2014). Another key to this translational effort will be the further development of selective kappa opioid PET imaging in normal and affected human subjects, which is still at a nascent stage. A more 'out of the box approach' is to take advantage of "creative" pharmacology. Buprenorphine is not only a mu partial agonist, but is a potent kappa antagonist having antidepressant activity (Karp et al, 2014). A recent open label clinical trial by Alkermes demonstrated that the nonselective KOR antagonist buprenorphine when combined with a mu opioid antagonist significantly reduced depressive symptoms in a population of individuals having treatment resistant depression (E. Ehrich, Kappa-2015 conference proceedings). Dr Andrew Saxon (Seattle, VA) also reported results from the NIDA-funded CURB study, which showed that while cocaine consumption was not significantly reduced by buprenorphine combined with a long acting mu antagonist, secondary analysis of the data from cocaine-using subjects showed a highly significant reduction in nicotine and ethanol use. Additional, recent findings in the dynorphin-kappa domain reported at the ' $3 r d$ Conference on the Therapeutic Potential of Kappa Opioids in Pain and Addiction' can be found at (http://depts.washington.edu/ nidactr/kappatherapeutics2015.html).

Human laboratory studies are an efficient means of bridging the gap between preclinical studies and clinical trials, and we encourage additional validations using more selective kappa antagonists, nevertheless, these early findings are provocative. In summary, the initial results using animal models of psychiatric diseases followed by early validation in human trials support the prediction that individuals unable to control their drug consumption because of overwhelming feelings of dysphoria or anxiety during the abstinence phase, may find kappa antagonists helpful by promoting stress resilience.

\section{FUNDING AND DISCLOSURE}

The authors declare no conflict of interest.

\section{ACKNOWLEDGMENTS}

This work is supported by United States Public Health Service Grants from the National Institute on Drug Abuse and the National Institute on Alcohol Abuse and Alcoholism.

\section{Charles Chavkin ${ }^{1}$ and George F Koob ${ }^{2}$ \\ ${ }^{1}$ Department of Pharmacology, University of Washington, Seattle, WA, USA; ${ }^{2}$ National Institute on Alcohol Abuse and Alcoholism, Rockville, MD, USA E-mail: cchavkin@u.washington.edu}

Bruchas MR, Land BB, Chavkin C (2010). The dynorphin/kappa opioid system as a modulator of stress-induced and pro-addictive behaviors. Brain Res 1314: 44-55.

Carroll FI, Carlezon WA Jr (2013). Development of $\kappa$ opioid receptor antagonists. J Med Chem 56: 2178-2195.

Karp JF, Butters MA, Begley AE, Miller MD, Lenze EJ, Blumberger DM et al (2014). Safety, tolerability, and clinical effect of low-dose buprenorphine for treatment-resistant depression in midlife and older adults. J Clin Psychiatry 75: e785-e793.

Koob GF, Buck CL, Cohen A, Edwards S, Park PE, Schlosburg JE et al (2014). Addiction as a stress surfeit disorder. Neuropharmacology 76 Pt B 370-382.

Lowe SL, Wong CJ, Witcher J, Gonzales CR, Dickinson GL, Bell RL et al (2014). Safety, tolerability, and pharmacokinetic evaluation of single- and multiple-ascending doses of a nove kappa opioid receptor antagonist LY2456302 and drug interaction with ethanol in healthy subjects. J Clin Pharmacol 54: 968-978.

Whitfield TW Jr, Schlosburg JE, Wee S, Gould A, George O, Grant Y et al (2015). к Opioid receptors in the nucleus accumbens shell mediate escalation of methamphetamine intake. J Neurosci 35 4296-4305.

Neuropsychopharmacology Reviews (2016) 41, 373-374; doi: I 0. I 038/npp.2015.258

\section{RiboTag: Not Lost in Translation}

Measuring RNA from a defined subset of cells derived from a complex tissue is an important challenge that has confounded the field. Two recently developed tools have simplified this issue. The RiboTag and BacTRAP (Translating Ribosome Affinity Purification) methods allow for immunoprecipitation of ribosome-associated RNA from specific cells within complex tissues by expressing tagged ribosomal protein in desired cell types (GFP-tagged RPL10 for TRAP and hemagglutinin-tagged RPL22 for RiboTag) (Doyle et al, 2008; Heiman et al, 2008; Sanz et al, 2009). More specifically, these methods allow analysis of the 'translatome'-ribosome-associated mRNA-which may be particularly sensitive to eventdependent regulation of protein translation. For example, RiboTag-expressing transgenic mice were recently used to compare differential gene expression responses to cocaine in striatal neurons expressing $\mathrm{D}_{1}$ and $\mathrm{D}_{2}$ dopamine receptors (Chandra et al, 2015). 